\title{
Pressure measurement in the cylinder of four-stroke marine engine - simulation analysis
}

The measurement of combustion pressure relies on connecting a pressure sensor to a four-stroke marine engine cylinder by a channel led out from the engine cylinder. The geometry of the channel distorts the results of combustion pressure measurements. The purpose of the work is to create a model of combustion processes in engine cylinders. The model uses a mathematical description of the indicator channel on one of the engine cylinders. The input data to the model and the data necessary for its verification came from the direct measurements on the research facility. The test object was a four-stroke Sulzer 3AL25/30 engine loaded by an electric power generator. During calculations, different diameters and lengths of measurement channel were simulated. The obtained results allowed to formulate the conclusion that the geometry of the measurement channel has a significant impact on the measurement results. The increase of the length of the measuring channel as well as the diameter of the channel causes disturbances in the measurement of the maximum pressure.

Key words: diesel engine, marine engine, combustion pressure, indicator channel

\section{Introduction}

Measurement of combustion pressure is a very important parameter in the diagnosis of marine Diesel engines. The measuring of combustion pressure allows detecting e.g.: a problem with piston rings leakage or a problem with fuel injection. Ship engines operate continuously for hundreds of hours, powering a ship or operating as an auxiliary engine on a ship that generates electrical energy. Therefore, proper diagnostics will enable early detection of a technical problem related to the proper functioning of the engine and avoid the withdrawal of the engine of operation as well as costly repairs.

However, measurement directly in the cylinder of the engine is often impossible due to the engine's construction as well as the high temperatures prevailing in the cylinder during engine operation. Mounting sensors that would be able to record cylinder pressure changes would increase engine costs.

Therefore, for measuring the combustion pressure in medium and low speed marine engines, a drainage channel is used from the piston engine cylinder ended with an indicator cock, later called the indicator channel. The main task of the channel is to remove water and impurities from the engine cylinder during initial engine rotation and blowdown. This way it protects the engines against damage caused by hydro-impact. During the engine operation, this channel is also used to measure the combustion pressure. The sensor is mounted on an indicator cock, i.e. at the end of the indicator channel. Such assembly causes the sensor to be removed from the cylinder. This assembly reduces the cost of combustion pressure measuring. However, the results of the pressure measurement show a discrepancy between the actual cylinder pressure and the pressure on the sensor in the indicator cock.

The shape of the indicator channel can cause discrepancies. Between the cylinder and the sensor on the indicator cock, the diameter of the channel changes as well as bends appear at different angles in the channel. The Kistler company, which produces piezoelectric pressure sensors, already indicates the problem of combustion pressure mea- surement, caused by the shape of the channel resulting from the natural frequency of the indicator channel. In order to correct the measurement carried out, they indicate that the use of the Bergh and Tijdeman model based on flow equations [1].

Engin Oezatay also mentions this problem of the actual measurement of the combustion pressure, but the reason for the distortion is the phenomenon of Helmholtz resonance occurring in the indicator channel [2].

The article aims to show the influence of the dimensions of the indicator channel to values of measured maximum combustion pressure. Special attention is paid to the maximum pressure in the indicator channel deviates from the value of the combustion pressure in the engine cylinder.

\section{Research facility}

The implementation of the work objective requires the selection of a research facility. The requirement for the test facility is the possibility of obtaining detailed data related to the geometry of the charge system and the fuel system. The facility consists of 3-cylinder A125/30 Sulzer engine and electric power generator. The engine is in the marine version. This means, among other things, that the engine cooling system is based on a high and low-temperature water circuit, with the low-temperature circuit being sea water, in this case the external water system, cooled by two cooling towers. The engine is charged by the VTR 160 BrownBoveri turbocharger and intercooled.

The fuel system consists of Bosch type injection pumps, controlled by the rotational speed governor through fuel racks and multi-hole fuel injectors. Fuel injectors are centrally located in the engine cylinder heads.

The AL25/30 engine is coupled with the GD8-500-50 generator which transmits the generated electrical energy to the water resistor. The engine is installed in the Maritime Laboratory of Gdynia Maritime University and operates at a constant rotational speed of $750 \mathrm{rpm}$.

The detailed parameters of the test facility are presented in Table 1 and Figure 1 [7]. 
Table 1. AL25/30 engine parameters [5]

\begin{tabular}{|l|c|c|}
\hline Parameter & Unit & Value \\
\hline Rotational speed & $\mathrm{rpm}$ & 750 \\
\hline Cylinder number & - & 3 \\
\hline Cylinder diameter & $\mathrm{mm}$ & 250 \\
\hline Stroke & $\mathrm{mm}$ & 300 \\
\hline Compression ratio & - & 12.7 \\
\hline Injection timing & ${ }^{\circ}$ before TDC & 18 \\
\hline
\end{tabular}

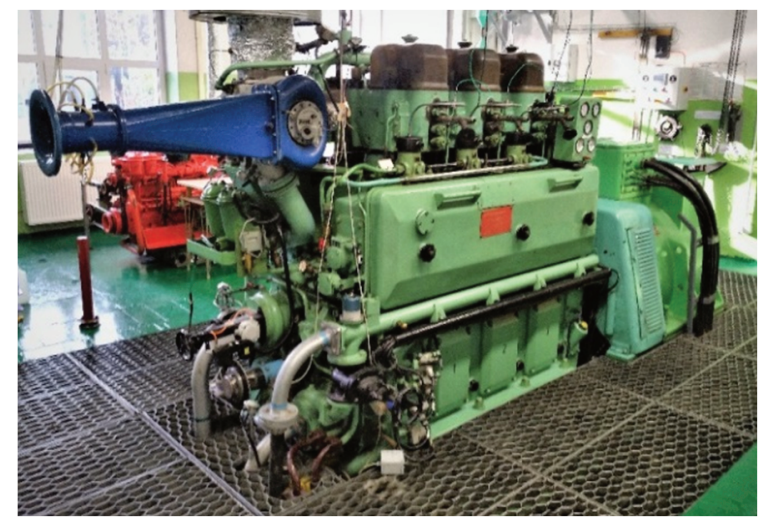

Fig. 1. Photo of the SULZER 3AL25/30 piston engine facility [7]

During the tests, the engine operated with a $250 \mathrm{~kW}$ load determined on the basis of the electrical output power from a generator. The measurement results were recorded in quasi-steady conditions.

During the tests, the combustion pressure was registered in all engine cylinders using Kistler 6613CG1 sensors coupled to a voltage amplifier. Each observation involved 7 revolutions of the crankshaft with a sampling rate of 720 measurements per crankshaft rotation.

\section{Assumptions of the model and its validation}

\subsection{Description of the model}

The main purpose of the work need to develop a combustion model of a marine piston engine and its verification based on the actual data of the 3AL25/30 engine.

This goal can be achieved by using a one-dimensional model. This model includes the following partial models: combustion process model, model of heat exchange in the engine cylinder construction elements, model of flows in air and exhaust channels, emission model of exhaust gases, mechanical friction model, model of phenomena occurring in the turbocharger and heat exchange model in the radiator [5].

Functional dependence between individual models is presented in Figure 2.

The $\mathrm{C} 1, \mathrm{C} 2$ and $\mathrm{C} 3$ are engine cylinders in which the combustion and heat exchange process was modelled. The model also includes mathematical descriptions of cylinder valve timing, piston movement, and direct fuel injection. The cylinders are connected to the pulse exchangers $\mathrm{J} 1$ and J2 via the exhaust ducts The exhaust gases flow through channels $6-8$ and 11 to the $T$ turbine and exit outside via channel 9.

The ambient air parameters are entered into the model at the SB1 and SB2 nodes. The air through the channel 1 is supplied to the compressor $\mathrm{C}$, then through the channel 2 to the air cooler CO1. After cooling, the air enters to engine cylinders via channels 3,4 and 5 .

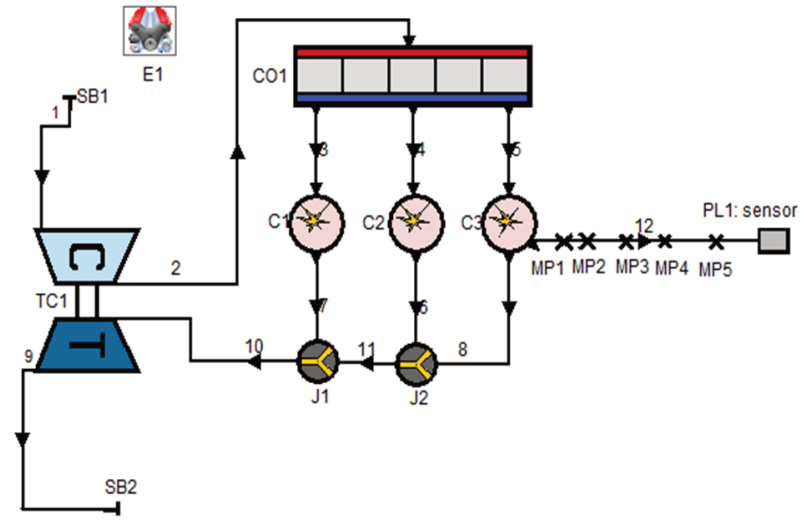

Fig. 2. Model of the research object in the AVL Boost program

The pipe number 12 it's the indicator channel MP symbols are the marked places then find the pressure sensor.

The E1 icon allows the basic engine and model parameters. The most important of them are:

- order of ignitions - in cylinders presented in the values of the rotation angle of the crank shaft, $0^{\circ}, 480^{\circ}$ and $240^{\circ}$ respectively,

- oil temperature - SAE10 equal to $67^{\circ} \mathrm{C}$ [5]

\subsection{Flow model}

The flow through all air channels was determined based on the isentropic flow equation, taking into account the mass inertia. In order to determine the mass flow rate, the geometric dimensions of the channels, the value of the isentropic exponent, temperature and pressure as well as the composition of the exhaust gas were needed. The geometrical dimensions were measured on the real object, while the initial values of thermodynamic parameters were adopted. In the case of air ducts as for the measured parameters after the air cooler, and for exhaust gases as for the measured gas parameters. The calculated thermodynamic parameters were determined using the Runge-Kutta iterative method [3].

It should be noted that the geometry of the exhaust gas channels behind the engine cylinders can contribute to the backflow of exhaust gases to adjacent cylinders. For this reason, these channels need to be routed to the collector collecting at an angle. In order to model this state of affairs, the so-called pulse exchangers (J1 and J2 in Fig. 2) are implemented.

Resistance movement in the channels was model using Equation 1:

$$
\frac{\mathrm{F}}{\mathrm{V}}=\varphi \frac{\lambda_{\mathrm{f}}}{2 \mathrm{~d}} \rho \mathrm{u}^{2}
$$

where: $\mathrm{F}$ - resistance $[\mathrm{N}], \mathrm{V}$ - volume $\left[\mathrm{m}^{3}\right], \varphi-$ Fanning coefficient of friction [-], value for the round section is $1, \lambda_{\mathrm{f}}$ - coefficient of resistance depending on the type of flow (laminar, mixed, turbulent), $\mathrm{d}$ - channel diameter $[\mathrm{m}], \mathrm{u}-$ flow speed $[\mathrm{m} / \mathrm{s}], \rho-$ gas density $\left[\mathrm{kg} / \mathrm{m}^{3}\right]$.

In all gas channels, there is also heat exchange with the environment through convection. The heat transfer coefficient for channels was determined by the Reynolds analogy method [4]. 


\subsection{Model validation}

The pressure sensor in the indicator channel on the real object is mounted as shown in Figure 3.

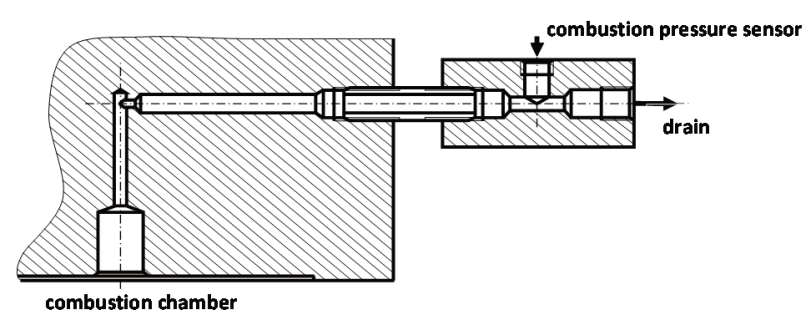

Fig. 3. The mounting position of the pressure sensor in the indicator channel

For the purpose of validation, the actual shape of the indicator channel was designed in the engine model compared to the results on the real object. Validation consisted in a comparison of measured and calculated values. According to the presented data, the majority of parameter values obtained by modeling do not differ from the measured values. The difference between the measured maximum pressure (Pmax) and the calculated by modeling was $0.94 \%$ and the position of the Pmax differs by $1 \mathrm{deg}$ of the crankshaft position.

\section{Analysis of results}

A positively valid model of the research object was used to analyse the phenomena taking place in the indicator channel. Results of the simulation on base on Eq. 1. are presented as follow. Figure 4 shows a model indicator channel in which points 100, 200, 300, 400 and $500 \mathrm{~mm}$ were used to calculate pressure changes in points MP1 to MP5. The length of L was $500 \mathrm{~mm}$ and $1000 \mathrm{~mm}$. Indicator channel was closed at the end of the indicator cock.

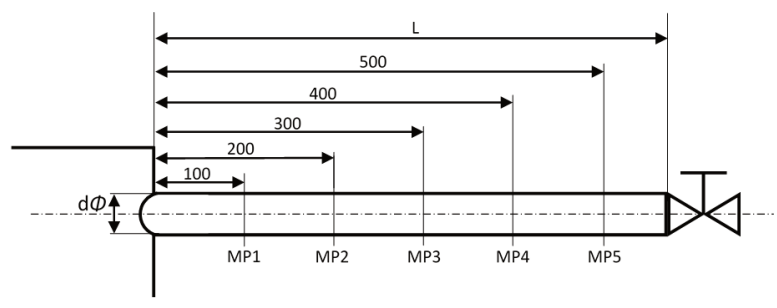

Fig. 4. Simulation indicator channel

Figure 5 shows a graph of pressure changes in the indicator channel in relation to the distance from the combustion chamber at diameters 5, 10, 15, 20, $25 \mathrm{~mm}$. It can be noticed that the calculated pressure increases in relation to the in-cylinder pressure by average $13.7 \%$. For the diameter of $5 \mathrm{~mm}$, the pressure decreases up to $300 \mathrm{~mm}$ from the cylinder and for the measuring places $400 \mathrm{~mm}$ and $500 \mathrm{~mm}$ increases.

It was decided to check whether the diameter of the indicator channel directly affects the combustion process. The upper side of Figure 6 presents graph of combustion pressure in the combustion chamber for various diameters of the indicator channel. The lower side of Figure 6 presents the dependence between differences between the combustion pressure in the cylinder and pressures obtained from meas- urement points and the diameter of the indicator channel. According to presented results of calculation, the highest combustion pressure occurs at the diameter of the $5 \mathrm{~mm}$ indicator channel. The increase of the indicator channel diameter causes observed decrease of the combustion pressure. Probably its effect of increase of the volume of combustion chamber by the volume of the indicator channel. The dependence presented on the lover side of Figure 6 shows that the distance of pressure sensor not more than $100 \mathrm{~mm}$ from the cylinder causes only little changes is the pressure measurement.

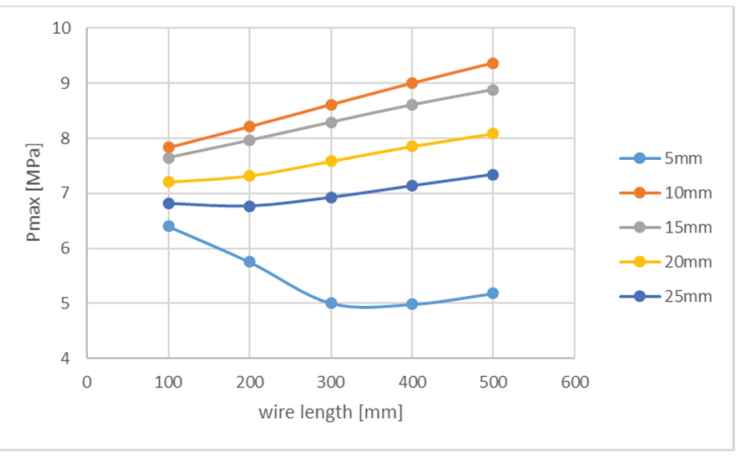

Fig. 5. Changes in the pressure in the indicator channel in relation to the distance from the combustion chamber
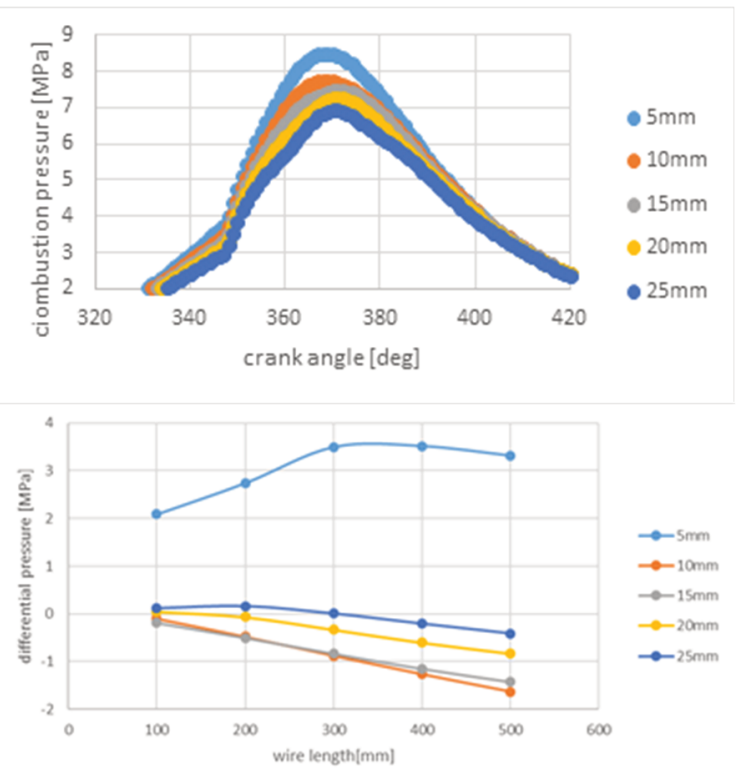

Fig. 6. The graph of combustion pressure in the combustion chamber with different diameters of the indicator channel (up) and a graph of the differences between the pressure in the combustion chamber and the indicator channel (down)

This result is convergent with conclusions presented by Kistler [1]. It should be noted, that the diameter of indication duct equals $5 \mathrm{~mm}$ causes significant decrease of the measured pressure. Moreover, its important that in the marine engines usually the length of indicator channel is longer than $100 \mathrm{~mm}$ and according to obtained results the increase of indicator duct length causes significant increase of the measured pressure. It means that long channel causes intensification of the wave phenomena in the channel. 
Then, the indicator channel was extended after the last MP5 calculation point by $500 \mathrm{~mm}$, thus the length of the indicator channel extended to $1000 \mathrm{~mm}$. Figure 7 shows a graph of pressure changes in the indicator channel with a diameter of $25 \mathrm{~mm}$ in relation to the distance from the combustion chamber.

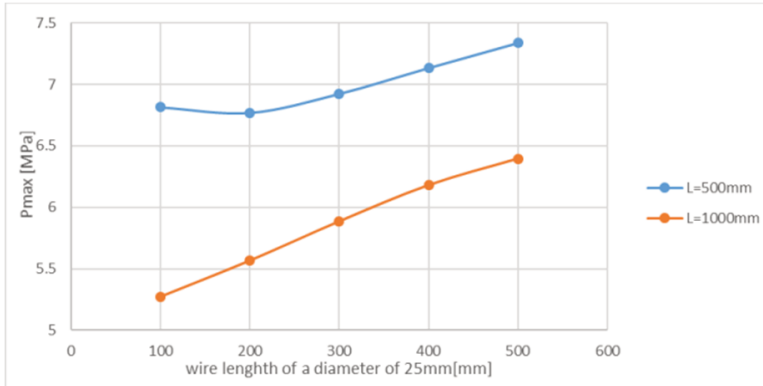

Fig. 7. Pressure changes in the indicator channel with a diameter of $25 \mathrm{~mm}$ in relation to the distance from the combustion chamber

It can be noticed that the decrease of the length of the indicator channel causes the decrease of the calculated pressure in the MP5 point. The maximum combustion pressure in a channel with a length of $500 \mathrm{~mm}$ increases in relation to the pressure in the cylinder by about $43.1 \%$ and at a length of $1000 \mathrm{~mm}$ by $24.7 \%$. This result prove the conclusion of possible influence of the wave phenomena on the measurement result.

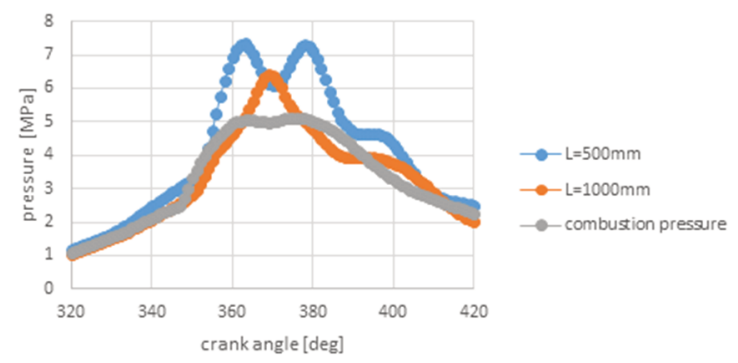

Fig. 8. Pressure at MP5 of the cylinder at different lengths of the indicator channel
Figure 8 shows the pressure diagram measured at 500 $\mathrm{mm}$ from the cylinder at different lengths of the indicator channel.

In the graph for a length of $500 \mathrm{~mm}$ it can be seen two local maxima of pressure. After extending the indicator channel to $1000 \mathrm{~mm}$ the local maximum of the pressure is only one. Both results are for the same combustion pressure in the engine cylinder. Grey graph present the example result of direct measurement of the pressure by the indicator channel with construction presented in Fig. 3.

\section{Conclusions}

The paper presents the results of calculations of the pressure in the indicator channel in a four-stroke marine engine, using CFD. According to the obtained results, the maximum pressure in the indicator channel deviates in relation to the value of the combustion pressure in the engine cylinder. In most cases, the pressure is even higher. Therefore, there is a possibility that in the indicator channel pressure accumulation occurs, possibly caused by wave phenomena, which are the effect of compressibility of gases. So it is necessary to pay special attention to the shape and dimensions of the indicator channel during the design.

It has been shown that the place where the sensor is installed along with the shape of the indicator channel is important in the diagnosis of the internal combustion engine. Improper installation location of the sensor and the construction of the indicator channel can significantly degrade the diagnostic information for the operator of the marine engine.

According to the results of the calculation of the dependence on the dimensions of the indicator channel, the value of the maximum pressure can range from $-21 \%$ to $+43 \%$ of the maximum combustion pressure. If the indicator channel is incorrectly designed or the sensor is installed in the wrong place, it may not be possible to determine the location of the maximum combustion pressure.

\section{Acknowledgements}

The project was supported by AVL Company according to University Partnership Program and license of AVL Boost software.

\section{Bibliography}

[1] WALTER, T. Application of an Improved Model for the Determination of Acoustic Resonances in Indicator Passages for Combustion Pressure Measurements in Large Bore Gas Engines. Kistler Instruments AG, Winterthur 2007.

[2] OEZATAY, E., ONDER, C. Model based sensor reconstruction of in cylinder pressure trace using indicator cock pressure information. CIMAC Congress 2010, 166, 1-9.

[3] BADER, P., MCLAREN, D., QUISPEL, G.R.W., WEBB, M. Volume preservation by Runge-Kutta methods. Applied Numerical Mathematics. 2016, 109, 123-137, DOI: 10.17863/ CAM.1184.

[4] INCROPERA, F.P., DEWITT, D.P. et al. Fundamentals of heat and mass transfer. John Wiley \& Sons, 2011.

[5] CUPER-PRZYBYLSKA, D. Model of processes of the AL25/30 marine engine and its verification. New Trends in Production Engineering, 2018, 1(1), 285-29, DOI: 10.2478/ ntpe-2018-0035.

[6] CUPER-PRZYBYLSKA, D. Przegląd modeli procesów spalania w cylindrze silnika tłokowego. Autobusy : technika, eksploatacja, systemy transportowe. 2018, 19(4), 48-52, DOI: 10.24136/atest.2018.020.

[7] CUPER-PRZYBYLSKA, D., WYSOCKI, J. Research injection pressure with the Kistler 4067E pressure transmitter on Sulzer engine 3AL25/30. Journal of KONES Powertrain and Transport. 2017, 24(3), 29-36, DOI: 10.5604/ 01.3001.0010.3061.

Dominika Cuper-Przybylska, MEng. - Faculty of Electrical Engineering, Gdynia Maritime University. e-mail: d.cuperprzybylska@we.umg.edu.pl

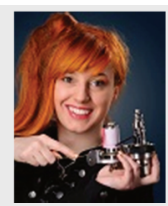

